Draft Version February 25, 2019

Preprint typeset using IATEX style emulateapj v. 6/22/04

\title{
TOPOLOGY OF STRUCTURE IN THE SLOAN DIGITAL SKY SURVEY: MODEL TESTING
}

\author{
J. Richard Gott, III ${ }^{1}$, D. Clay Hambrick ${ }^{1}$, Michael S. Vogeley ${ }^{2}$, Juhan Kim ${ }^{3}$, Changbom Park ${ }^{3}$, \\ Yun-Young $\mathrm{Choi}^{3}$, Renyue Cen ${ }^{1}$, Jeremiah P. Ostriker ${ }^{1}$, and Kentaro Nagamine ${ }^{4}$ \\ Draft version February 25, 2019
}

\begin{abstract}
We measure the three-dimensional topology of large-scale structure in the Sloan Digital Sky Survey (SDSS). This allows the genus statistic to be measured with unprecedented statistical accuracy. The sample size is now sufficiently large to allow the topology to be an important tool for testing galaxy formation models. For comparison, we make mock SDSS samples using several state-of-the-art N-body simulations: the Millennium run of Springel et al. (2005)(10 billion particles), Kim \& Park (2006) CDM models (1.1 billion particles), and Cen \& Ostriker (2006) hydrodynamic code models (8.6 billion cell hydro mesh). Each of these simulations uses a different method for modeling galaxy formation. The SDSS data show a genus curve that is broadly characteristic of that produced by Gaussian random phase initial conditions. Thus the data strongly support the standard model of inflation where Gaussian random phase initial conditions are produced by random quantum fluctuations in the early universe. But on top of this general shape there are measurable differences produced by non-linear gravitational effects (cf. Matsubara 1994), and biasing connected with galaxy formation. The N-body simulations have been tuned to reproduce the power spectrum and multiplicity function but not topology, so topology is an acid test for these models. The data show a "meatball" shift (only partly due to the Sloan Great Wall of Galaxies; this shift also appears in a sub-sample not containing the Wall) which differs at the $2.5 \sigma$ level from the results of the Millennium run and the Kim \& Park dark halo models, even including the effects of cosmic variance.
\end{abstract}

Subject headings: cosmology:observations - large-scale structure of universe - methods:numerical

\section{INTRODUCTION}

The topology of large scale structure in the universe is an important physical property of the matter density field that can be compared with the prediction of the simple inflationary models (Guth 1981; Linde 1983) where Gaussian random phase initial conditions are generated from quantum fluctuations in the early universe. Analytic tools for quantitatively analyzing the topology of large scale structure in three dimensions have been developed during the past 20 years (Gott, Melott, \& Dickinson 1986; Hamilton, Gott, \& Weinberg 1986; Gott, Weinberg, \& Melott 1987, Gott et al. 1989; Vogeley, et al. 1994; Park, Kim, \& Gott 2005; Park et al. 2005). The distribution of galaxies in space is smoothed to construct isodensity contour surfaces whose topology may be computed. Our genus statistic - described below - quantifies the topology of isodensity contours.

On smoothing scales larger than the correlation length, the fluctuations are still in the linear regime and since fluctuations in the linear regime grow in place without changing topology, the topology we measure now should reflect that of the initial conditions, which should be of Gaussian random phase according to the theory of inflation (Gott. Weinberg \& Melott 1987). We have shown this in detail by comparison with large N-body simulations (Gott. Weinberg \& Melott 1987). We expect sponge-like topology at the median density contour to

\footnotetext{
${ }^{1}$ Princeton University Observatory, Princeton, NJ 08544

2 Department of Physics, Drexel University, Philadelphia, PA 19104

3 Korea Institute for Advanced Study, CheongNyangNi, Seoul 130-722, Korea

${ }^{4}$ Department of Physics, University of Nevada, Las Vegas, 89154 Electronic address: dclayh@astro.princeton.edu
}

be a strong prediction of inflation (Gott, Melott, and Dickinson 1986, Gott, Weinberg, Melott 1987). Previous models of galaxy clustering suggested either a meatball topology - isolated clusters growing in a low density connected background as suggested by the Press and Schechter (1974) formalism or by hierarchical galaxy formation (Peebles 1974, Soneira \& Peebles 1978) - or a Swiss cheese topology - isolated voids surrounded on all sides by walls as suggested by Einasto, Joeveer, \& Saar (1980). But with Gaussian random phase initial conditions we expect a sponge-like topology as pointed out by Gott. Melott. \& Dickinson (1986).

Studies of many observational samples have been conducted by our group and others, which have shown in every case a sponge-like median density contour as expected from inflation. For notable examples, see Gott, Melott, \& Dickinson (1986), Gott et al. (1989), Moore et al. (1992), Vogeley et al. (1994), Canaveses et al. (1998), Hikage et al. (2002), Hikage et al. (2003), and Park et al. (2005). In addition, in all cases the observed genus curve was reasonably well-fit by the Gaussian random phase theoretical curve in equation 4 . Perhaps the most spectacular such agreement was seen in the Caneveses et al. (1998) analysis of the 15,000 galaxy PSCz redshift survey. This study showed quite a good fit (within the noise) to the random phase curve at a variety of smoothing lengths. The IRAS galaxies in this sample are primarily low mass spiral and irregular galaxies and so may suffer less biasing effects than galaxies from an optically selected sample. See Park et al. (2006) for discussion of the strong dependence of morphological fraction on density in the SDSS.

A two-dimensional variant of the genus statistic can also be applied to redshift slices, sky maps, and CMB 
maps (Coles 1988, Melott et al. 1989, Gott et al. 1990 , Park et al. 1998), In this case, $G(\nu)=\#$ of hot (or high density) spots - \# of cold (or low density) spots, and the Gaussian random phase hypothesis implies $g(\nu) \propto$ $\nu \exp \left(-\nu^{2} / 2\right)$. All of these studies (Redshift Slices: Park et al. 1992; Colley 1997; Hoyle, Vogeley, \& Gott 2002; Hoyle et al. 2002; Sky Maps: Gott, Mao, Park, \& Lahav 1992; Park, Gott, \& Choi 2001; CMB Maps: Smoot et al. 1994; Colley, Gott, \& Park 1996; Kogut et al. 1996; Colley \& Gott 2003; Park 2003; Spergel et al. 2006; Gott et al. 2006) indicate consistency with the Gaussian random phase hypothesis. The CMB maps are a particularly powerful test of the Gaussian random phase hypothesis because the fluctuations are still firmly in the linear regime. The dramatic agreement between the 2D CMB results and the Gaussian random phase hypothesis strongly supports idea of the standard theory of inflation and that the initial conditions were truly Gaussian random phase.

The three-dimensional topology data on galaxy clustering are particularly interesting because they allow us not only to confirm their general Gaussian random phase nature (on large scales), but also to test for non-linear processes and bias involved with galaxy formation. Matsubara (1994) has discussed how the genus curve may be altered by second-order non-linear gravitational clustering effects, which can show up at small smoothing lengths. Vogeley et al. (1994) explicitly measured the diminution of the genus amplitude caused by these effects. Park, Kim, \& Gott (2005) have studied other important alterations which can occur by non-linear gravitational evolution, redshift space distortion, and biasing associated with galaxy formation.

In this paper we compute genus curves for volumelimited samples of the largest galaxy redshift survey to date, the Data Release 5 (DR5) of the SDSS, and for mock samples from state-of-the-art N-body simulations. Our goal is to examine whether the models for galaxy formation represented by these simulations are consistent with the observations. This is a potentially powerful test, because the input parameters of the flat $\Lambda \mathrm{CDM}$ model used in these simulations were determined by fitting to a host of other observations - CMB anisotropy data, large-scale power spectrum and correlation function of galaxies, SNeIa luminosity-distance data, cluster abundances and baryon fraction, etc.- -but not topology of large-scale structure in the galaxy distribution. As the basic underpinnings of the model become more secure, we can turn to more precise testing of models for the physics of galaxy formation. Likewise, the methods and parameters for simulating galaxies have been tuned to match other observations, but not topology. Thus, this comparison provides an independent test of the model for structure formation.

\section{THE GENUS AND RELATED STATISTICS}

The genus is a measure of the topology of the large scale distribution of galaxies. We first smooth the point distribution of galaxy positions (we use only volumelimited samples in the analysis below) with a Gaussian smoothing ball of radius $\lambda$

$$
W(r)=\frac{1}{(2 \pi)^{3 / 2}} e^{-r^{2} / 2 \lambda^{2}},
$$

where $\lambda$ is chosen to be greater than or equal to the correlation length. In this paper we choose $\lambda=6 h^{-1} \mathrm{Mpc}$ which is approximately equal to the galaxy correlation length. This smallest scale yields the highest resolution measure of the three-dimensional topology and the greatest statistical power because of the large number of resolution elements. This scale also gives the greatest amount of information about non-linear gravitational effects and biasing involved in galaxy formation.

We establish density contour surfaces labeled by $\nu$, where the volume fraction on the high density side of the density contour surface is $f$ :

$$
f=\frac{1}{\sqrt{2 \pi}} \int_{\nu}^{\infty} e^{-x^{2} / 2} d x
$$

The genus as a function of $\nu$ is given by

$$
G(\nu)=\# \text { of donut holes }- \text { \# of isolated regions }
$$

(Gott, Melott, and Dickinson, 1986). Thus, an isolated cluster has a genus of -1 by this definition. We have shown that $G(\nu)$ is also equal to minus the integral of the Gaussian curvature over the area of the contour surface divided by $4 \pi$, which enables us to measure the genus with a computer program (CONTOUR 3D) (see Gott, Melott, \& Dickinson 1986 and Gott, Weinberg, \& Melott 1987).

For a Gaussian random phase density field, the genus per unit volume, $g(\nu) \equiv G(\nu) / V$, is given by

$$
g(\nu)=A\left(1-\nu^{2}\right) e^{-\nu^{2} / 2},
$$

where the amplitude $A=\left(\left\langle k^{2}\right\rangle / 3\right)^{3 / 2} /\left(2 \pi^{2}\right)$ depends only on the average value of $k^{2}$ integrated over the smoothed power spectrum (Hamilton, Gott, \& Weinberg 1986; Adler 1981; Doroshkevich 1970; Gott, Weinberg, \& Melott 1987). Thus, the amplitude $A$ [units: genus $\left./\left(h^{-1} \mathrm{Mpc}\right)^{3}\right]$ can tell us about the primordial power spectrum. For a Gaussian random field, the median density contour ( $\nu=0, f=50 \%$ volume enclosed) exhibits a sponge-like topology (many holes and no isolated regions); the $f=7 \%$ high density contour $(\nu=1.5)$ shows isolated clusters, while the $f=93 \%$ density contour $(\nu=-1.5)$ is dominated by isolated voids. We call the curves $G(\nu)$ and $g(\nu)$ (which differ only by a constant factor for a given sample) the "genus curves".

For the purpose of examining departures of the observed genus curve from the Gaussian random phase prediction, we parameterize the genus curve by several derived quantities. First is the best-fit amplitude,

$$
A=\text { amplitude of the genus curve, }
$$

which we measure by least squares fit of the theoretical random phase curve to the data, fitting only in the range $-1<\nu<1$. For the random phase case, this amplitude is proportional to $\left(\left\langle k^{2}\right\rangle^{3 / 2}\right)$ of the smoothed power spectrum and so gives information about the primordial power spectrum. For observations, this amplitude appears lower because of non-linear clustering and biasing due to coalescence of structures (Park \& Gott 1991b; Vogeley et al. 1994; Canavezes et al. 1998)

We quantify shifts and deviations of the genus curve from the shape of the random phase curve using the following three variables. We measure horizontal shifts of 
the central part of the genus curve with

$$
\Delta \nu=\frac{\int_{-1}^{1} g(\nu) \nu d \nu}{\int_{-1}^{1} g_{\mathrm{rf}}(\nu) d \nu},
$$

where $g_{\mathrm{rf}}(\nu)$ is the genus of the random phase curve following the formula in equation 4 using the fitted amplitude $A$ above. The theoretical curve (Eq. (4) has $\Delta \nu=0$. A negative value of $\Delta \nu$ is called a "meatball shift" as it is caused by a greater prominence of isolated connected high-density structures which push the genus curve to the left. A positive value of $\Delta \nu$ is called a "bubble shift" as it can be caused by a greater prominence of isolated voids, and might be produced by isolated explosions (Ostriker and Cowie 1981) as opposed to inflation. A slight, statistically significant "meatball shift" $(\Delta \nu<0)$ was observed first by Gott et al. (1989), who examined the CfA, Giovanelli \& Haynes, and Tully datasets. In hindsight, one can see a slight "meatball shift" in the very first genus curve ever measured (Gott, Weinberg, Melott 1987) and this "meatball shift" was also seen for brighter galaxies in an analysis of an earlier sample of the SDSS (Park et al. 2005). This shift is presumably due to nonlinear galaxy clustering and bias associated with galaxy formation.

To quantify departures of the observed genus from the random phase prediction in the region of the genus curve where isolated voids should dominate, we measure

$$
A_{v}=\frac{\int_{-2.2}^{-1.2} g(\nu) d \nu}{\int_{-2.2}^{-1.2} g_{\mathrm{rf}}(\nu) d \nu},
$$

where $g_{\mathrm{rf}}(\nu)$ is again the genus of the best fit random phase curve following the formula in equation 4 (see Park. Kim. \& Gott 2005 for an explanation of the choice of range in $\nu$ ). As shown in Park. Kim, \& Gott (2005), a value of $A_{v}<1$ can be the result of biasing in galaxy formation because voids are very empty and can coalesce into a few larger voids. $A_{v}$ is sensitive to the number of isolated voids but the density contour (at $\nu=-1.7$ for example) is given by the volume fraction, so if $A_{v}$ is less than 1 , and by definition there is the same volume in the low density regions being measured, there must therefore be fewer but larger voids. Non-linear clustering alone at these scales predicts a value of $A_{v}>1$ for the power spectrum of the $\Lambda C D M$ model we adopt (see Figure 1 of Park, Kim, \& Gott 2005), so observing $A_{v}<1$ may be an indication of bias in galaxy formation.

Similar to $A_{v}$, we measure a quantity $A_{c}$ that characterizes departure from random phase behavior in the part of the genus curve expected to be sensitive to the number of isolated high-density regions (clusters),

$$
A_{c}=\frac{\int_{1.2}^{2.2} g(\nu) d \nu}{\int_{1.2}^{2.2} g_{\mathrm{rf}}(\nu) d \nu} .
$$

A value of $A_{c}<1$ may occur because of non-linear clustering, when clusters collide and merge. Also if there is a single large connected structure like the Sloan Great Wall, this can also lower the value of $A_{c}$. Also, as Park, Kim, \& Gott (2005) have shown, the Matsubara (1994) formula for second-order gravitational non-linear effects has the result that $A_{v}+A_{c}=2$ at all scales, so if we observe both $A_{v}$ and $A_{c}$ to be less than 1, (as we find below to be the case) biased galaxy formation must be involved.

\section{N-BODY SIMULATIONS OF LARGE-SCALE STRUCTURE}

Before we confront results of current simulations of the flat $\Lambda \mathrm{CDM}$ model with the best observations of the topology of the galaxy distribution currently available, it is instructive to consider the remarkable success to date of large N-body simulations in modeling large-scale structure. It is encouraging that as the volume and resolution in N-body simulations have grown with the size and quality of observational data sets, that the agreement has become even more spectacular-perhaps a sign that we are on the right track with these models.

Peebles did the first large N-body simulation for cosmology using 1000 dark matter particles with $\Omega_{m}=1$ and Poisson initial conditions. It showed clusters like the Coma cluster forming from random fluctuations by gravitational instability and a reasonable covariance function. Aarseth, Gott and Turner (1973) used 4,000 particles with initial conditions that had more power on large scales than Poisson (index $n=-1$ ). They found power law covariance functions quite like those observed even for models with $\Omega_{m}<1$ and $n=-1$ (Gott, Turner, \& Aarseth 1979, Gott \& Turner 1979) as originally proposed theoretically by Gott \& Rees (1975). (Indeed, inflationary flat lambda models popular today have $\Omega_{m}<1$ and more power on large scales than Poisson initial conditions, just as these early simulations suggested.) They also found voids as large as those observed. The largest voids had volumes such that at the mean density they would have contained as much mass as the Coma type clusters contained. This was reasonable from theoretical considerations of non-linear clustering, considering cluster (Gunn and Gott 1972) and void (Bertschinger 1985, Fillmore \& Goldreich 1984) formation from small fluctuations via gravitational instability. In Gaussian random phase initial conditions, isolated over- and under-dense regions in the initial conditions should be equal in mass leading to equal mass great clusters and empty voids lacking the same amount of mass. They also found that such $\Omega_{m}<1$ models with more power at large scales than Poisson produced better multiplicity functions than $\Omega_{m}=1$ Poisson models (Gott \& Turner 1977; Bhavsar, Gott, \& Aarseth 1981).

The advent of inflation brought for the first time realistic theoretical power spectra to input into N-body models. Together, inflation and Cold Dark Matter specified reasonable initial conditions. A suite of such simulations by Davis et al. (1985) provided an impressive match to many aspects of the observed large-scale structure.

Just as theory seemed to be converging on the nowdisproven "standard CDM model," the observations provided a shock. De Lapparent, Geller \& Huchra (1986) found many voids $50 h^{-1} \mathrm{Mpc}$ across. This caused a number of people to abandon Gaussian random phase initial conditions and gravitational instability - favoring explosions to produce the voids instead (Ostriker \& Cowie 1981). Then Geller \& Huchra (1989) discovered the CfA Great Wall of galaxies, which surprised everyone. This result was announced at an IAU conference in Rio de Janeiro. Many people said that was the end for random phase initial conditions, for one expected the covariance function to die at a scale of about $30 h^{-1} \mathrm{Mpc}$ and here was a structure that was $150 h^{-1} \mathrm{Mpc}$ long. "Perhaps it was produced by cosmic string wakes," was one comment 
made at the time (though not by us).

However, the jump to abandon random phase initial conditions ignored the fact that no N-body simulations had been done by that time that were large enough to properly model structures as large as the CfA Great Wall. When Park (1990) did such simulations using 4 million particles, simulating such a volume for the first time, the results showed that such great walls form routinely. In fact, a $20^{\circ}$ thick slice survey through the simulations was a near perfect visual match to the Geller and Huchra survey. These simulations used a standard peak biasing scheme and included both standard CDM and $\Omega_{m}=0.4$ models. Narrow $6^{\circ}$ thick slices showed prominent large voids like those in the De Lapparent, Geller \& Huchra slice and great walls appeared in $20^{\circ}$ thick slices. This simulation showed weak narrow walls and filaments of galaxies inside the voids, as seen in the CfA data (Park, Gott, Melott, \& Karachentsev 1992).

In similar fashion, N-body dark matter simulations large enough to mimic the deep pencil beam surveys of Broadhurst, Ellis, Koo, \& Szalay (1990) showed apparently regular spikes (walls) of galaxies just like those observed (Park \& Gott 1991a). And large N-body simulations (Park 1991) showed great attractors just like that observed by Lynden-Bell et al. (1988). Great repulsors are not seen because such peaks in the gravitational potential occur in the middle of large voids where there are too few tracer galaxies. N-body simulations including hydrodynamics have been successful in modeling the Lyman Alpha forest (Cen, Miralda-Escude, Ostriker, \& Rauch 1994; Hernquist, Katz, Weinberg, \& MiraldaEscude 1996).

Prior to this study we did an analysis of the topology of a large N-body computer simulation made to mimic the Sloan Digital Sky Survey (Colley et al. 2000). This 54 million particle simulation was observed from one location to simulate what will be seen by the Sloan Digital Sky Survey, to produce sky maps, slices, and 3D topology maps, to show the power of the survey. The sky map looked astonishingly like real sky maps made to similar depth, and the slice maps looked quite like similar survey maps made in the Las Campanas Survey (Kirshner et al. 1981) and now seen in the SDSS. The cosmological model for this simulation was the flat $\Lambda \mathrm{CDM}$ model which remains in favor.

Even larger simulations are available today and we are interested to see how they fare in their ability to model the topology of large-scale structure. The "Millennium Run" (MR hereafter), using over 10 billion $\left(2160^{3}\right)$ dark matter particles (Springel et al. 2005) and surveying a cube of side length $500 h^{-1} \mathrm{Mpc}$, has shown structures remarkably like the Great Wall found by Geller \& Huchra (1989), and even wall complexes somewhat resembling the Sloan Great Wall which Gott et al. (2006) measured to span 1.37 billion light years (Springel, Frenk \& White 2006). Indeed Figure 1 in Springel et al. (2006) shows a remarkable visual agreement between what is seen in the Millennium Run and in slices of the CfA, the 2dF survey and the SDSS. The most noticeable difference is that the Sloan Great Wall looks much more visually prominent and coherent than the longest chain of walls found in the MR. (In this simulation, the box size of $500 h^{-1}$ Mpc cuts off the power spectrum at larger scales. If a simulation were to be made with a larger box size, it would have more power at these larger scales and therefore could more easily produce large coherent structures like the Sloan Great Wall.) The MR computes dark matter halo formation merger trees and uses a semi-analytic model to simulate the galaxy-formation process where star formation and feedback are modeled by simple analytic physical models. Croton et al. (2006) have produced mock galaxy samples of their cube that include galaxies brighter than the Magellanic clouds, including absolute magnitudes on the SDSS system, which allow us to make mock SDSS galaxy samples.

Park, Kim \& Gott (2005) produced 8.6 billion particle $\left(2048^{3}\right)$ simulations that cover volumes of $\left(1024 h^{-1} \mathrm{Mpc}\right)^{3}$ and $\left(5632 h^{-1} \mathrm{Mpc}\right)^{3}$. These simulations employ a Halo Occupation Distribution (HOD) method to place an appropriate number of galaxies in heavy halos identified by the PSB (Kim \& Park 2006) and FoF techniques. These simulations were used to analyze the effects of galaxy formation and bias on topology by Park, Kim, \& Gott (2005). More recently, Kim \& Park have produced 1.1 billion particle simulations covering a volume of $\left(614 h^{-1} \mathrm{Mpc}\right)^{3}$. Here they use a new technique to identify physically bound dark matter halos (not tidally disrupted by larger structures) at the present epoch and identify these with galaxies (we call these the DH simulations, for Dark (matter) Halos). They too have produced magnitudes for these mock galaxies on the SDSS system by matching the halo mass function with the luminosity function of the SDSS galaxies.

Cen \& Ostriker (2006) have run hydrodynamic simulations covering a smaller cube of $\left(120 h^{-1} \mathrm{Mpc}\right)$ on a side using an 8.6 billion $\left(2048^{3}\right)$ cell hydro mesh, with $\left(1024^{3}\right)$ dark matter particles. Here the galaxy formation process is simulated with a hydrodynamic code that identifies collapsing regions, calculates star formation rates, and includes radiative cooling/heating, UV background radiation with local attenuation, and supernova feedback associated with star formation. Again, some assumptions about star formation are made, but this model has one of the most detailed and direct physical calculations of the galaxy formation process available for any simulation that spans a cosmologically-interesting volume. For further details of the simulation, we refer the readers to Cen. Nagamine \& Ostriken (2005). Nagamine has produced the mock catalogs giving absolute magnitudes on the SDSS system from the Cen \& Ostriker (2006) hydro simulations.

The MR simulation, the Kim \& Park DH simulation and the Cen \& Ostriker hydro simulation represent state-of-the-art simulations for different schemes to mimic galaxy formation. While the astronomical community seems to be converging on a standard model for cosmology - the flat $\Lambda$ CDM model (see, e.g., Reiss et al. 1998, Perlmutter et al. 1999, de Bernadis et al. 2000, and Spergel et al. 2006) - galaxy formation remains an unsolved problem. This means that since only one cosmological model need be simulated, larger N-body runs exploring different galaxy formation scenarios from different teams can be run. Since the parameters in the semianalytic models have been tuned to account for other features such as covariance function and multiplicity function, and topology was not considered, topology is a particularly stringent test. If the models produce the right topology automatically, it would constitute dramatic ev- 
idence that their galaxy formation scenarios were on the right track. In any case, a successful model must show the universe in all its features, including topology.

\section{SLOAN DIGITAL SKY SURVEY DATA}

The SDSS (York et al. 2000; Stoughton et al. 2002; Adelman-McCarthy et al. 2006) is a survey to explore the large scale distribution of galaxies and quasars by using a dedicated 2.5m telescope (Gunn et al. 2006) at Apache Point Observatory. The photometric survey has imaged roughly $\pi$ steradians of the Northern Galactic Cap in five photometric bandpasses denoted by $u, g, r, i$, and $z$ centered at $3551,4686,6165,7481$, and $8931 A$, respectively, by an imaging camera with 54 CCDs (Fukugita et al. 1996; Gunn et al. 1998). The limiting magnitudes of photometry at a signal-to-noise ratio of $5: 1$ are $22.0,22.2,22.2,21.3$, and 20.5 in the five bandpasses, respectively. The median width of the PSF is $1.4^{\prime \prime}$, and the photometric uncertainties are 2\% RMS (Abazajian et al. 2004). See Ivezic et al (2004) for details of assessment of photometric quality and Tucker et al. (2006) for discussion of the monitor telescope pipeline employed for calibration.

After image processing (Lupton et al. 2001; Stoughton et al. 2002; Pier et al. 2003) and calibration (Hogg et al. 2001; Smith et al. 2002), targets are selected for spectroscopic follow-up observation. The spectroscopic survey is planned to continue through 2008 as the Legacy survey and yield about $10^{6}$ galaxy spectra. The spectra are obtained by two dual fiber-fed CCD spectrographs. The spectral resolution is $\lambda / \Delta \lambda \sim 1,800$, and the RMS uncertainty in redshift is $\sim 30 \mathrm{~km} / \mathrm{s}$. Because of the mechanical constraint of using fibers, no two fibers can be placed closer than $55^{\prime \prime}$ on the same tile. Mainly due to this fiber collision constraint, incompleteness of the spectroscopy survey reaches about 6\% (Blanton et al. 2003a) in such a way that regions with high surface densities of galaxies become less prominent even after adaptive overlapping of multiple tiles. This angular variation of sampling density is accounted for in our analysis.

The SDSS spectroscopy yields three major samples: the main galaxy sample (Strauss et al. 2002), the luminous red galaxy sample (Eisenstein et al. 2001), and the quasar sample (Richards et al. 2002). The main galaxy sample is a magnitude-limited sample with apparent Petrosian $r$-magnitude cut of $m_{r, \lim } \approx 17.77$ which is the limiting magnitude for spectroscopy. It has a further cut in Petrosian half-light surface brightness $\mu_{\mathrm{R} 50 \text {,limit }}=24.5$ $\mathrm{mag} / \operatorname{arcsec}^{2}$. More details about the survey can be found on the SDSS web site ${ }^{5}$.

In our study, we use a subsample of SDSS galaxies known as the New York University Value-Added Galaxy Catalog (NYU-VAGC; Blanton et al 2005). This sample is a subset of the recent SDSS Data Release 5. One of the products of the NYU-VAGC used here is LargeScale Structure sample DR4plus (LSS-DR4plus). We use galaxies within the boundaries shown in Figure 1 of Park et al. (2006), which improves the volume-to-surface area ratio of the survey (important when smoothing). There are also three stripes in the Southern Galactic Cap observed by SDSS. Density estimation is difficult within these narrow stripes, so we do not use them.

\footnotetext{
5 http://www.sdss.org/dr5/
}

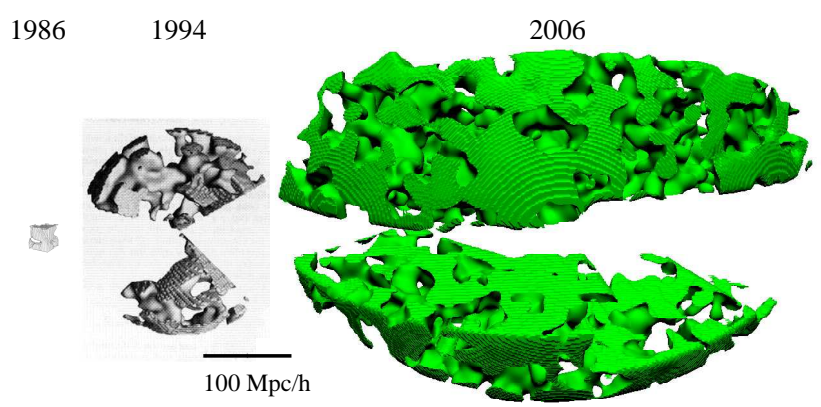

FIG. 1. - 50\% high volume contours from three galaxy surveys across three decades. From left to right, they are Gott, Melott, \& Dickinson (1986), Vogeley et al. (1994), and the present work.

The remaining survey region covers $4,471 \mathrm{deg}^{2}(1.362$ steradians). The primary sample of galaxies used here is a subset of the LSS-DR4plus sample referred to as void0, which is further selected to have apparent magnitudes in the range $14.5<r<17.6$ and redshifts in the range $0.001<z<0.5$. These cuts yield a sample of 312,338 galaxies. The roughly $6 \%$ of targeted galaxies which do not have a measured redshift due to fiber collisions are assigned the redshift of their nearest neighbor.

Completeness of the SDSS is poor for bright galaxies with $r<14.5$ because of both the spectroscopic selection criteria (which exclude objects with large flux within the three arcsecond fiber aperture; the cut at $r=14.5$ is an empirical approximation of the completeness limit caused by that cut) and the difficulty of obtaining correct photometry for objects with large angular size. For these reasons, analyses of SDSS galaxy samples have typically been limited to $r>14.5$; using the magnitude limits of the void0 sample, the range of absolute magnitude is only 3.1 at a given redshift.

The comoving distance and redshift limits of the volume-limited sample we analyze are determined from absolute magnitude limits obtained by using the formula

$$
m_{r, \lim }-M_{r, \lim }=5 \log ((1+z) r)+25+\bar{K}(z)+\bar{E}(z),
$$

where $\bar{K}(z)$ is the mean $K$-correction, $\bar{E}(z)$ is the mean luminosity evolution correction, and $r$ is the comoving distance corresponding to redshift $\mathrm{z}$. We adopt a flat $\Lambda$ CDM cosmology with density parameters $\Omega_{\Lambda}=0.73$ and $\Omega_{m}=0.27$ to convert redshift to comoving distance. To determine sample boundaries we use a polynomial fit to the mean $K$-correction,

$$
\begin{aligned}
\bar{K}(z)= & 3.0084(z-0.1)^{2} \\
& +1.0543(z-0.1)-2.5 \log (1+0.1) .
\end{aligned}
$$

We apply the mean luminosity evolution correction given by Tegmark et al. (2004), $E(z)=1.6(z-0.1)$. The rest-frame absolute magnitudes of individual galaxies are computed in fixed bandpasses, shifted to $z=0.1$, using Galactic reddening corrections (Schlegel 1998) and $K$ corrections as described by Blanton et al. (2003b). This 
means that a galaxy at $z=0.1$ has a $K$-correction of $-2.5 \log (1+0.1)$, independent of its SED.

From this sample, we construct a volume-limited sample containing galaxies brighter than absolute magnitude $M_{r}=-20.2$ and fainter than $M_{r}=-21.7$, and spanning comoving distance from $171.3 h^{-1} \mathrm{Mpc}$ to $344.5 h^{-1} \mathrm{Mpc}$ (corresponding to $z=0.0578-0.1178$ ). This observational sample is similar to the BEST sample studied in Park et al. 2005, but now larger in extent.

This volume-limited sample contains 70,781 galaxies (before overlap correction) and has a mean galaxy separation of $6.097 h^{-1} \mathrm{Mpc}$, so we can safely apply a Gaussian smoothing length of $6 h^{-1} \mathrm{Mpc}$. Numerous numerical experiments have shown that if the smoothing length is smaller than $1 / \sqrt{2} \simeq 0.71$ times the mean inter-galaxy separation there can be a "meatball shift" due to the algorithm picking out individual galaxies as isolated high density regions (Gott, Weinberg, \& Melott 1987, Gott et al. 1989). In this sample the smoothing length is approximately equal to 0.98 times the mean interparticle separation so this shot noise effect should be small. In any case, this is not critical for our analysis because we compare the observations directly with mock galaxy catalogs from N-body simulations. These mock catalogs are constructed to cover exactly the same range in absolute magnitude as seen in the observations and so contain very nearly (within a few percent) the same total number of galaxies in the sample. Because the techniques being applied to the observations and the N-body simulation mock catalogs are identical, the results should be identical (within statistical variation) if the N-body simulations are correctly modeling the distribution of galaxies.

\section{TOPOLOGY OF LARGE-SCALE STRUCTURE IN THE SDSS}

In a previous paper (Park et al. 2005) we analyzed the three-dimensional topology of large-scale structure in the SDSS at a range of smoothing lengths and compare this with theoretical expectations. In the present paper we focus on results with a smoothing length of $6 h^{-1} \mathrm{Mpc}$, which yields the most resolution elements and gives the most important information on galaxy formation. The sample of galaxies available has now grown significantly larger and so we are now able to make direct comparison of this sample with large N-body simulations and their various methods of modeling of galaxy formation. As we shall see, the observational sample is now large enough that the topology, as measured by the genus curve $g(\nu)$, is now a powerful tool for testing models of galaxy formation.

Figure 1 shows the progression by date of survey of the 3D topology of selected galaxy redshift surveys. All have similar smoothing lengths of $5-6 h^{-1} \mathrm{Mpc}$ and show the median density contour surface with the high density regions shown as solid and the low density regions as empty. According to standard inflationary theory this median density contour should be spongelike. The small cube on the left shows the $3 \mathrm{D}$ region studied by Gott et al. (1986). The earth is at the lower front right corner of the cube. The topology is spongelike and the Virgo cluster is included in the high density region. The larger region of isodensity contours in the center of this figure is from the the CfA redshift survey (Vogeley et al. 1994).

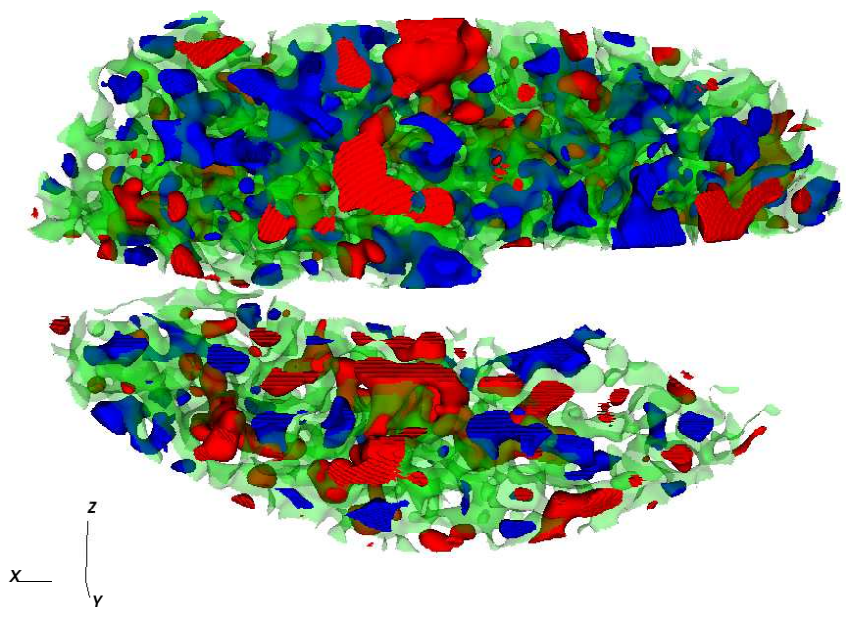

FIG. 2.- $7 \%$ low (blue) $50 \%$ (green), and $7 \%$ high (red) volume contours in our SDSS sample. The Sloan Great Wall is visible as the long red structure in the lower slice.

The earth is at the center and the upper fan shaped region is in the North Galactic Hemisphere while the lower fan shaped region lies within the South Galactic Hemisphere. The Great Wall noted by Geller \& Huchra (1989) can be seen connecting high density regions across the top fan-shaped region. Again the topology is spongelike, with the high density regions all connected together, and the low density regions also connected in an interlocking pattern.

Finally, the portion of the SDSS data now available (in 2006, a full twenty years after the first figure) is shown on the right of Figure 11 This is the largest region yet studied for topology and contains nearly 400,000 galaxies in total. The location of the earth is at the back. The horizontal slice extending out toward us is the northern equatorial slice of the SDSS and includes the Sloan Great Wall (Gott et al. 2005). The upper slice is a second contiguous thick region in the northern hemisphere of the SDSS. (When SDSS-II is complete in 2008, the gap between these two slices will be filled in.) It is easy to see that the topology of this median density contour is spongelike. The high density regions (taking up half the volume) form one multiply connected region (shown solid) and the low density regions (taking up the other half of the volume) also form one multiply connected region that is interlocking with the high density region.

Figure 2 shows the same regions of the SDSS, but with isodensity contours in different colors for different volume fractions. The $7 \%$ high density regions - containing the highest density $7 \%$ of the volume - are solid red. The end of the Sloan Great Wall can be seen as the red structure snaking from the left to the right in the Equatorial slice. This red contour also shows isolated high density regions (clusters) as expected from the random phase genus curve. The $50 \%$ high density contour is shown in transparent green - this contour is a multiply connected spongelike surface that divides the high density half of the sample from the low density half. The $7 \%$ low density regions are shown as solid blue and show isolated voids. The red and blue regions lie on opposite sides of the transparent green spongelike surface. 

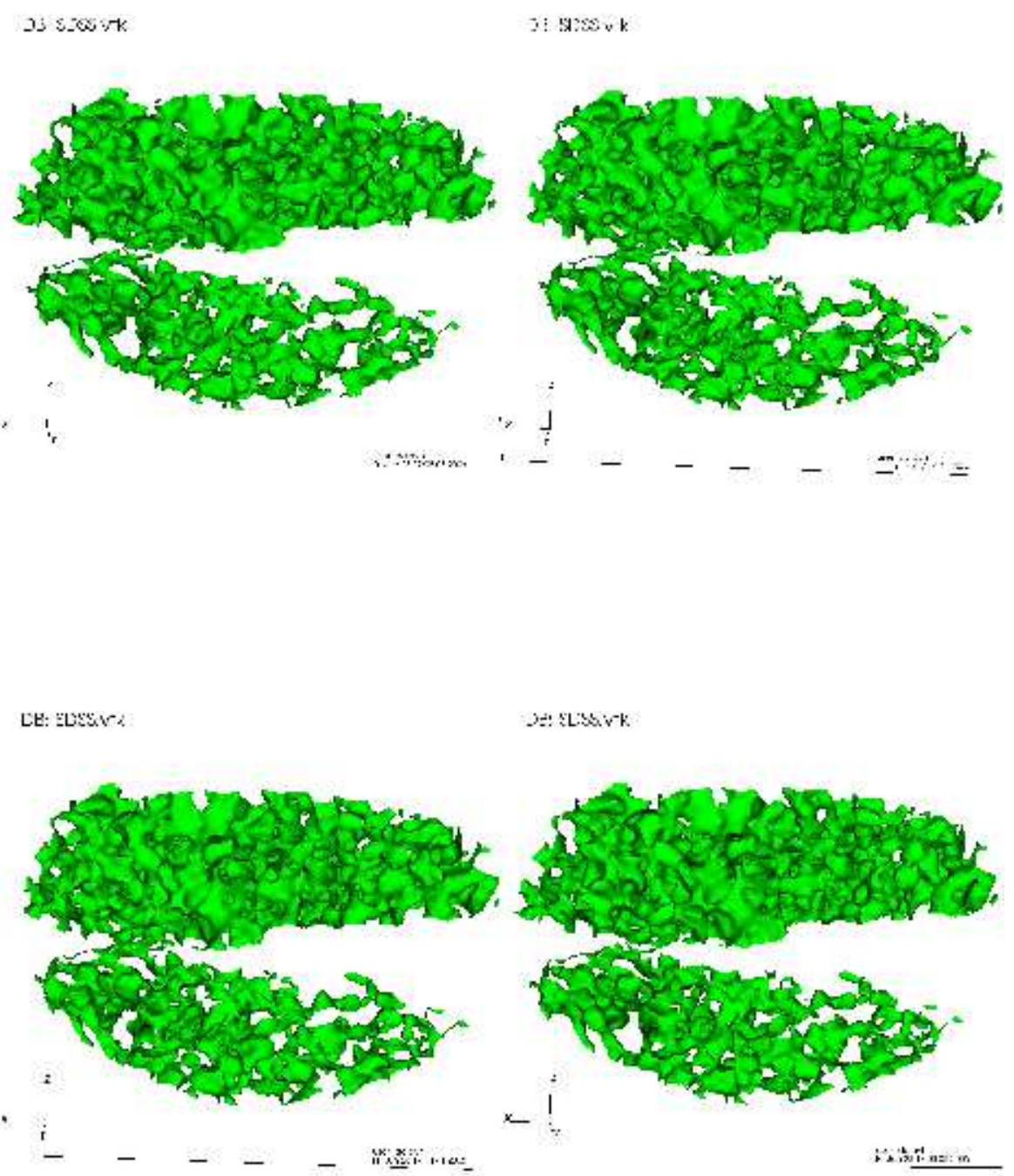

FIG. 3.- Uncrossed (upper) and crossed (lower) images of the 50\% density contour for our sample of the SDSS. These images are displayed with separation equal to the separation between your eyes. To view the upper pair, touch your nose to the page between the two views. The pictures will look blurry but each stereo view will be directly in front of the proper eye. Slowly raise your nose from the paper keeping the blurry images fused into one blurry stereo view. As you back away to reading distance you will be able to bring these two fused views into focus so that you can see the 3D image clearly. (A stereo viewer may also be used.) To see the lower pair, cross your eyes by looking at an object (finger or pen tip) held in front of the page, moving it until you see three images, then shift your gaze to the central image.

To facilitate viewing the three-dimensional nature of the density contours, Figure 3 shows a stereo pair of the same SDSS contours. This offers our best picture yet of the $3 \mathrm{D}$ topology of large scale structure in the universe.

Figure 4 shows the genus curve of this volume-limited SDSS sample smoothed at $\lambda=6 h^{-1} \mathrm{Mpc}$. In this figure we compare the observed genus curve with results for mock surveys produced from the N-body simulations, which we describe in Section 6 below. Also shown in Figure 4 is the random phase genus curve that best fits the SDSS genus curve. The data approximately follow this random phase curve, as expected from inflation. How- ever, there are measurable departures that are likely to have been caused by non-linear effects and galaxy formation. We characterize these differences from the random phase curve using the measures $A, \Delta \nu, A_{v}$, and $A_{c}$ described above and plot these values in Figures 5 and 6

Figure[5]shows the $(\Delta \nu, A)$ plane with the SDSS values of $(\Delta \nu, A)$ plotted as a solid blue square, while the black circle indicates the random phase prediction of $\Delta \nu=0$ (the amplitude of this point is not that of a random phase distribution, but rather is fixed to be the same as the SDSS data).

The two blue X's show the values computed from the 


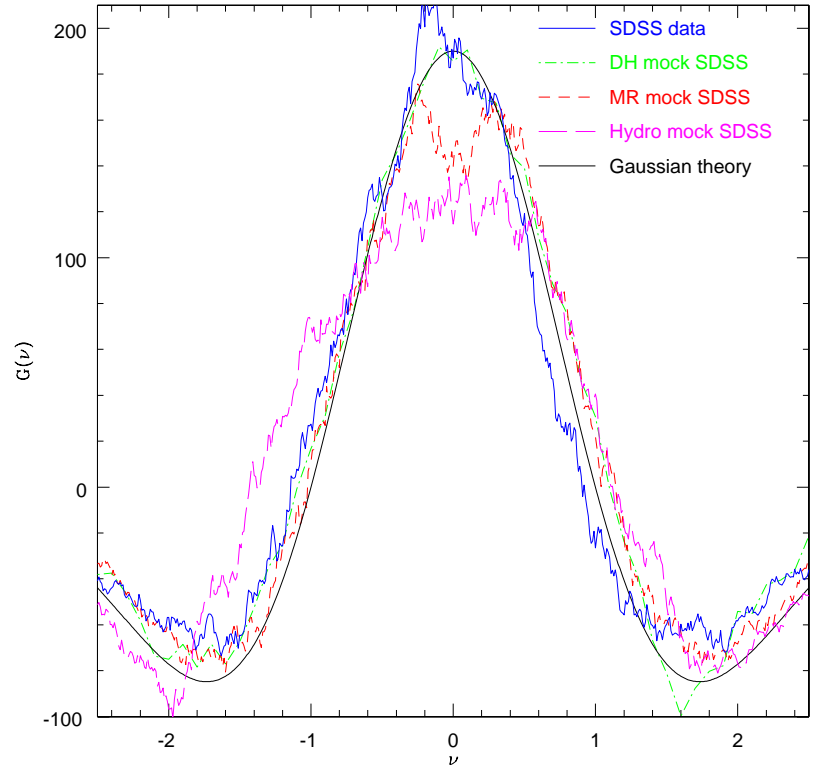

FIG. 4.- Genus curves for the SDSS sample, hydro sample, and random samples drawn from the $100 \mathrm{DH}$ and $50 \mathrm{MR}$ studies. The Gaussian random curve is shown for comparison. Notice that we plot $G(\nu)$ (i.e., do not divide by the sample volume) in order to show the genus of the entire sample at each $\nu$.

two SDSS slices separately - as if each were the only sample studied. In this case, the median density contour is calculated for each sub-sample separately, thus each has a somewhat different density level at the median contour because presence of the Sloan Great Wall in the equatorial sample guarantees that the median density in this sample is greater than in the northern sample. The difference between the genus parameters of these two subsamples provides a rough measure of the cosmic variance. The values of the genus amplitude, $A$, for the two subsamples are of course smaller, which we correct for by dividing each by the respective fraction of the entire volume it represents.

The observed genus curve in Figure 4 is displaced slightly to the left in the central regions (a slight meatball shift, or a prominence of clusters over voids). This gives the curve a negative value of $\Delta \nu$ as indicated by equation 6] The whole sample has a value of $\Delta \nu=-0.08$. The SDSS Great Wall itself is a very prominent simplyconnected high density region and so by itself can cause a meatball shift. Indeed, the equatorial sample which contains the Sloan Great Wall has a value of $\Delta \nu=-0.11$. However the northern sample which does not contain the Sloan Great Wall has by itself a value of $\Delta \nu=-0.08$. Thus, the meatball shift in the data is a general phenomenon and is not due solely to the Sloan Great Wall.

Such a meatball shift has been seen in observational samples before. It was first noticed and commented on by Gott et al. (1989) who examined the CfA, Giovanelli \& Haynes, and Tully samples. Gott, Cen, and Ostriker (1996) found that hydrodynamic simulations predicted a meatball shift for (early type) elliptical galaxies relative to (late type) spiral galaxies (elliptical galaxies tend to congregate more in isolated rich clusters), an effect

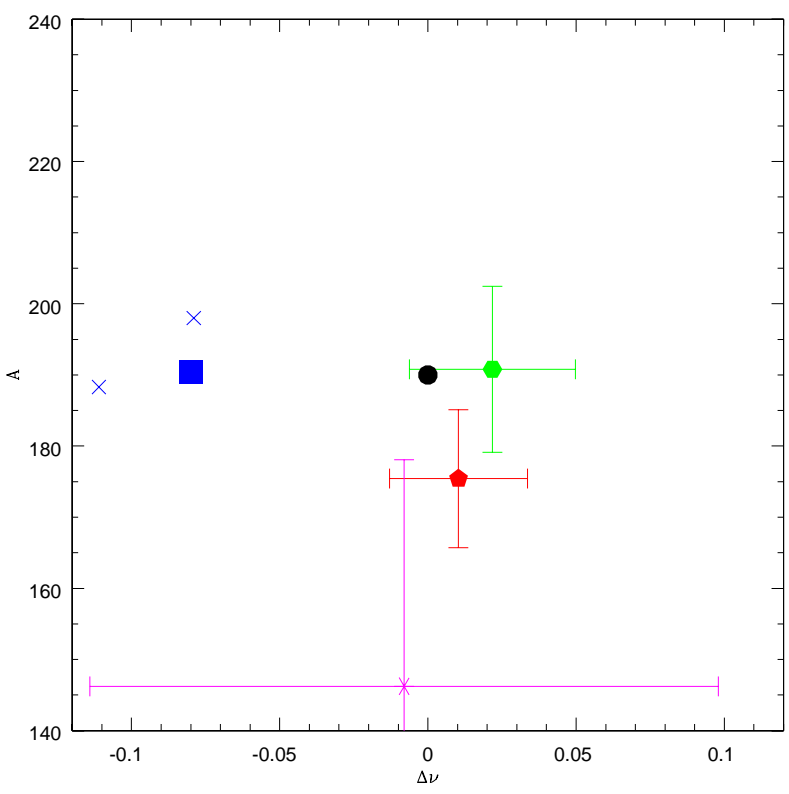

FIG. 5.- Plot of the genus shift parameter $\Delta \nu$ versus the genuscurve amplitude $A$ for our samples. The black circle corresponds to Gaussian random phase. The blue square is the SDSS sample, with the two blue Xs representing each of the two SDSS subregions (normalized in amplitude to the volume of the whole sample). The green hexagon is the mean of the $100 \mathrm{DH}$ mock Sloans, with error bars representing the standard deviation of that sample. The red pentagon is the mean of the $50 \mathrm{MR}$ mock Sloans, with error bars showing that standard deviation. The pink $\mathrm{X}$ denotes the hydrodynamic simulation of Cen \& Ostriker; the error bars correspond to the cosmic variance of $\left(120 h^{-1} \mathrm{Mpc}\right)^{3}$ subregions within a $\left(500 h^{-1} \mathrm{Mpc}\right)^{3}$ box.

later observed in the 2D topology analysis of the SDSS by Hoyle et al.(2002) where a meatball shift in red (early type) galaxies was seen relative to blue (late type) galaxies. Thus, it is clear that galaxy formation processes can produce meatball shifts relative to the random phase curve, as we observe with high statistical significance. The question is whether our galaxy formation models accurately reproduce this effect. Below we discuss whether they successfully model this shift in the genus curve.

Figure 6 shows the $\left(A_{v}, A_{c}\right)$ plane with the SDSS data again shown as a solid blue square, with the random phase prediction shown as a black circle. Again, X's indicate measurements for the two regions of the SDSS considered separately. The data have values of $\left(A_{v}, A_{c}\right)$ that depart from the random phase values of $(1,1)$ due to biased galaxy formation and non-linear effects.

\section{TOPOLOGY OF SDSS DATA VS. SIMULATIONS}

The genus curve for the SDSS sample shows a clear shift towards a "meatball" topology and behavior in the void and cluster-dominated tails that indicate fewer isolated voids and clusters than expected from either a Gaussian random phase distribution or from perturbation theory (see Matsubara 1994 and Park et al. 2005). In this section we examine whether current simulations of large-scale structure reproduce these features and use the simulations to assess the statistical significance of these departures from random phase behavior.

Our approach is to construct mock SDSS redshift sam- 
TABLE 1

Genus Statistics FOR SDSS AND Simulations theREOF

\begin{tabular}{|c|c|c|c|c|}
\hline Name & Amplitude & $\Delta \nu$ & $A_{v}$ & $A_{c}$ \\
\hline SDSS & 190.46 & -0.080 & 0.747 & 0.804 \\
\hline DH & $190.79 \pm 11.65$ & $0.022 \pm 0.028$ & $0.806 \pm 0.052$ & $0.811 \pm 0.067$ \\
\hline MR & $175.42 \pm 9.69( \pm 9.86)$ & $0.010 \pm 0.023( \pm 0.036)$ & $0.845 \pm 0.057( \pm 0.081)$ & $0.862 \pm 0.063( \pm 0.097)$ \\
\hline Hydro & $146.23[ \pm 31.9]( \pm 31.9)$ & $-0.008[ \pm 0.106]( \pm 0.107)$ & $0.783[ \pm 0.328]( \pm 0.328)$ & $1.016[ \pm 0.218]( \pm 0.219)$ \\
\hline
\end{tabular}

Note. - Errors not in parentheses are the standard deviations of the population in question (not given for the real SDSS or the Hydro simulation, where there is only one sample); errors in parentheses additionally include the effect of cosmic variation within a $1024 h^{-1} \mathrm{Mpc}$ box, and the bracketed errors for Hydro represent cosmic variation for its box size within a $500 h^{-1} \mathrm{Mpc}$ box.

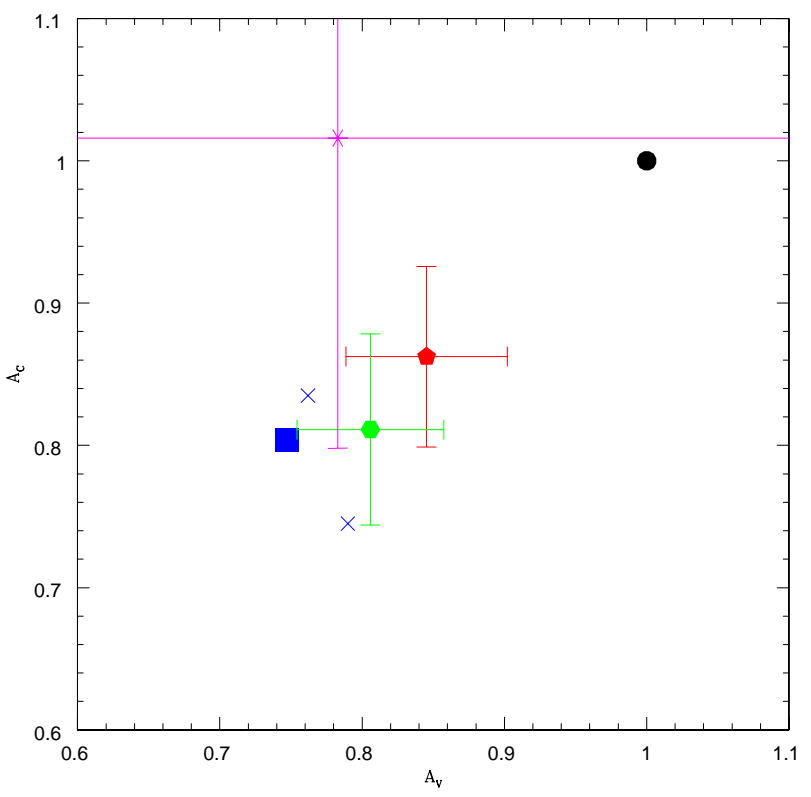

FIG. 6. - The same as Fig. 5 plotting $A_{v}$ and $A_{c}$. The upper-left blue X corresponds to the SDSS region without the Sloan Great Wall.

ples from each of the simulations that mimic the observational selection effects caused by the survey geometry, sampling density of structure, and redshift-space distortions. In the $\mathrm{DH}$ and MR cases, we construct many such mock surveys, smooth and compute the genus curves, and compute the variables $A, \Delta \nu, A_{v}$, and $A_{c}$ for each. Then we compute the mean and standard deviations of those statistics, as plotted in Figures 5 and 6 .

In considering the predictions of the various N-body simulations it is important to estimate the cosmic variance one may encounter. We start with the DH simulation. This simulation has a volume of $\left(614 h^{-1} \mathrm{Mpc}\right)^{3}$, which is a bit over 16 times the volume of the SDSS sample, allowing roughly that many independent mock surveys. We create 100 such surveys of the SDSS in order to fully sample the structure in the cube with the irregular shape of the SDSS; they are clearly not independent, but the mean and distribution function of the genus statistics are not affected by this. We show the mean and standard deviations of the genus quantities for the Park et al. simulations in Figures [5] and [6] with a green hexagon and associated uncertainty limits. It is clear that the SDSS is more than $3 \sigma$ away from the mean value of $\Delta \nu$. In fact none of the 100 mock surveys has a value of $\Delta \nu$ as negative as the observations. It could be argued that the SDSS contains the SDSS Great Wall, which is so unusual that this region should be excluded from the analysis. However, this is exactly the purpose of the 100 mock surveys: to examine the range of values we expect in SDSS-sized surveys. So the fact that the SDSS is outside the 2 sigma error bars from the cosmic variance expected shows that the DH simulations are not successful in predicting the observed meatball shift. Also, recall that the values of $\Delta \nu$ for both SDSS sub-samples (one not containing the Sloan Great Wall) are outside these limits.

The DH simulations are perfect in amplitude relative to the data. Importantly, the DH simulations (as well as the Millennium Run and the Cen and Ostriker simulations) include the effects of baryon oscillations which give the correct initial power spectrum. A similar DH simulation with 8.6 billion particles but without baryon oscillations had an amplitude of $209 \pm 11$, which is $1.6 \sigma$ high relative to the data (191). This shows that the topology can detect the presence of the baryon oscillations by measuring the slope of the power spectrum-simulations without them produce an amplitude of the genus curve that is too high. The values of $\Delta \nu=+0.020 \pm 0.027$ for those simulations without the baryon oscillations were not appreciably different from the ones that include the baryon oscillations.

For the MR simulation, we have a volume of $\left(500 h^{-1}\right.$ $\mathrm{Mpc})^{3}$ which enables us to make roughly 10 independent mock surveys of the SDSS. Again, to fully sample the cube, we make 50 mock surveys at random position and orientation. The mean and standard deviation of these mock surveys are shown in Figures [5] and [6] as a red pentagon with associated error bars. This indicates the cosmic variance seen from SDSS-sized mock surveys drawn from the $\left(500 h^{-1} \mathrm{Mpc}\right)^{3}$ survey region.

However, we must additionally consider the effect of cosmic variance on the scale of the simulation region: error bars on simulations with smaller box sizes will be somewhat underestimated with respect to larger (e.g., the MR w.r.t. the $\mathrm{DH}$ ), and with respect to the real universe (which of course has infinite box size). To estimate this added variance, we take 8 sub-cubes of volume $\left(512 h^{-1} \mathrm{Mpc}\right)^{3}$ out of the larger DH simulation of Kim \& Park (2007, in prep.) mentioned above, of volume $\left(1024 h^{-1} \mathrm{Mpc}\right)^{3}$, and make redshift maps of them; i.e., 
we give the galaxies their correct $x$ and $y$ coordinates, but put them at a $z$ coordinate equal to $z+v_{z} H_{0}^{-1}$ as if we were viewing a redshift space map of the survey region from a great distance. (Note that even with the different power spectrum, the variance of the genus statistics should still be correct.) We then compute the 3D topology for each of the 8 sub-cubes and measure the standard deviation of the 8 mean values of the parameters $(\Delta \nu, A)$ and $(A v, A c)$. This cosmic variance of the 8 sub-cubes is added to the variance of the $\mathrm{DH}$ sample population (i.e., the standard deviations are added in quadrature) to produce an effective standard deviation we expect to find due to cosmic variance for SDSS-sized surveys in the MR if we had a larger $\left(1024 h^{-1} \mathrm{Mpc}\right)^{3}$ simulation from which to draw mock catalogs. The increase in the standard deviation is only a few percent for the amplitude $A$ but $\sim 50 \%$ for the other three genus statistics (see Table 1 for both sets of values). We would have liked to make a similar set of sub-cubes for the $\left(614 h^{-1} \mathrm{Mpc}\right)^{3}$ DH simulation, but of course one cannot draw a statistically significant number of samples of that size from a $\left(1024 h^{-1} \mathrm{Mpc}\right)^{3}$ box without significant overlap.

The fact that the MR has a smaller volume also produces a systematic effect in that it has less power in its power spectrum at large scales (no power beyond $\left.\left(500 h^{-1} \mathrm{Mpc}\right)^{3}\right)$ so we should expect its amplitude $A$ to be systematically a little large. Actually, the amplitude is lower than for the $\mathrm{DH}$ simulations and is about $1 \sigma$ low relative to the data so apparently the effects of cosmic variance in a sample this size are more important than the systematic effect caused by the lack of longwavelength modes.

For the Cen and Ostriker hydro simulation we have only one $\left(120 h^{-1} \mathrm{Mpc}\right)^{3}$ simulation volume. This is about one-eighth the volume of the SDSS region so using its periodic boundary conditions we simply replicate it to make a volume large enough to encompass the SDSS. We then make a mock redshift catalog with the same absolute magnitude limits. Because the periodicity kills the large scale power we expect the simulation to be choppier than the real universe and therefore have a higher amplitude $A$ than the observations. But even more importantly, we expect a large cosmic variance in a sample only as small as $\left(120 h^{-1} \mathrm{Mpc}\right)^{3}$. To model this we construct 64 sub cubes each of volume $\left(120 h^{-1} \mathrm{Mpc}\right)^{3}$ from the MR simulation (of volume $\left.\left(500 h^{-1} \mathrm{Mpc}\right)^{3}\right)$, and as above we make redshift maps of them (giving galaxies their correct $x$ and $y$ coordinates but put them at a $z$ coordinate equal to $\left.z+v_{z} H_{0}^{-1}\right)$. Then we compute the $3 \mathrm{D}$ topology for each of the 64 sub cubes and measure the standard deviation of the 64 values of the parameters $(\Delta \nu, A)$ and $\left(A_{v}, A_{c}\right)$. This cosmic variance from the 64 sub cubes produces the error bars surrounding the one value from the Cen et al. simulation (shown as a magenta $\mathrm{X}$ with error bars.) We may then add the extra cosmic variance as per the MR data point to produce the standard deviations corresponding to a volume of $\left(1024 h^{-1}\right.$ $\mathrm{Mpc})^{3}$ (again given in Table 1 the extra st. dev. is $\lesssim 1 \%$ for all statistics). These error bars are very large; this one simulation is not very constraining of the topological properties. Roughly speaking, the volume of the Cen and Ostriker simulation is an eighth that of the SDSS so we expect error bars that are roughly $\sqrt{8} \simeq 2.8$ times as large as for the cosmic variance seen in the SDSS. This ratio is approximately correct as Figures [ [ [ [ show.

In Figure 4 we show the genus curve for the Cen and Ostriker simulation. It is not a good fit to the SDSS observations. The top of the genus curve is chopped off, and it has a meatball shift in the void region that is not seen in the observations. In the central region $-1<\nu<1$, where $\Delta \nu$ is measured, the curve is too fat, but also has a small negative value of $\Delta \nu=-0.01$. This is more negative than either the MR or the DH simulations, but is still not very close to the observed value of $\Delta \nu=-0.08$. The amplitude of the Cen and Ostriker simulation is much lower than the other three data sets. Overall the Cen et al. simulation does not give a good fit to the data; however, the volume of the simulation is small and the large error bars show that the observations are within 2 sigma in both $\Delta \nu$ and $A$. (To be fair, other hydro simulations by Cen and his collaborators - for example, Gott, Cen, \& Ostriker 1996 - have produced good looking genus curves, so this one may be suffering from a bit of bad luck.)

So, currently, the Cen and Ostriker hydro simulations are too small. They need to be increased in volume by about a factor of 10 to be fairly tested against the SDSS. In 1975 the largest N-body cosmological simulation had 4,000 particles (Aarseth, Gott, \& Turner 1975). In 1990 the largest N-body cosmological simulation had 4 million particles (Park 1990). This led Gott to predict in 1990 that by 2005 the record would be 4 billion particles. Springel et al. (2005) did even better than this with over 10 billion particles. An increase of a factor of 10 every 5 years is just what would be predicted by Moore's law (a doubling every 18 months). So given Moore's law we should expect that Cen and his colleagues will have a hydro code simulation with volume 10 times larger by 2011. Then we can see if it outperforms the MR or the DH simulations. Just one additional hydro run with the same parameters would also be interesting - would it be closer to the observations (suggesting some bad luck in the current run) or further away? With the current error bars, the MR, DH simulations, and the Cen and Ostriker simulations are all consistent with each other within $2 \sigma$.

In Figure 6] we compare the results for $A_{v}$ and $A_{c}$. Here the observations and the Millennium Run and the DH simulations are in better agreement. The observations have values of $\left(A_{v}, A_{c}\right)=(0.75,0.80)$ which depart from those of the random phase genus curve $(1,1)$. As we have mentioned, values of $A_{v}<1$ on these scales are not produced by non-linear gravitational clustering but must be produced by biased galaxy formation (Park, Kim \& Gott 2005). Here the Millennium run and the DH simulations are both moved from the random phase value in the direction of the observations. The $\mathrm{DH}$ simulation does better, being nearly perfect in $A_{c}$ and just over one sigma away in $A_{v}$. The Millennium Run is within one sigma in $A_{c}$ and around $1.7 \sigma$ away in $A_{v}$ (neglecting cosmic variance). Again, the Cen and Ostriker simulation is further away in $A_{c}$, but its larger error bars leave it within 1 sigma in both $A_{v}$ and $A_{c}$.

A possible issue with all these simulations is that their initial conditions assume a value of $\sigma_{8}=0.9$ at the present epoch to be consistent with the fit for the WMAP first-year data (Spergel et al. 2003), but the WMAP three-year data prefer a lower value of $\sigma_{8}=0.76$ (Spergel 
et al. 2006). This implies less non-linear growth of clustering up to the present epoch, and more biasing. We can estimate the possible effect of using a smaller value of $\sigma_{8}$ by examining the genus statistics at a slightly earlier epoch in a simulation designed to reach linear $\sigma_{8}=0.9$ at $z=0$. For example, at $z=0.5$ (when $\sigma_{8}=0.76$ ) in the DH simulations, we find that dark matter halos with the same density as the $z=0$ halos have $\Delta \nu=0.015$, versus $\Delta \nu=0.02$. Compared to the discrepancy of $\Delta \nu$ between the SDSS data and simulations, this is an infinitesimal effect and we conclude that this does not appear to be a problem. We reach similar conclusions about the effect of $\sigma_{8}$ on $A_{v}$ and $A_{c}$. The small size of these effects over this range of redshift (and so, over this same range of $\sigma_{8}$ ) can also be seen in Figure 4 of Park et al. (2005).

Another small but interesting effect has to do with halo or galaxy identification: nearby halos (or galaxies in the hydro simulation) may get merged together and only counted as one point. To estimate this effect, we calculated the genus of SDSS after merging together all galaxies closer than 100kpc (an overestimate of the actual scale of the problem). This does seem to move SDSS slightly closer to the simulations: the amplitude decreased by $\sim 0.5 \%, \Delta \nu$ increased from -0.080 to -0.078 , and $A_{v}$ increased by $\sim 1 \%$, while $A_{c}$ decreased by about the same amount.

Figure 4 shows the genus curve of the observations versus one of the 50 MR mock surveys picked at random, one of the $100 \mathrm{DH}$ mock surveys picked at random, and the hydro mock survey. The DH simulation looks the best. The top of the genus curve near $\nu=0$ is not cut off and it looks most like the observations. Both the Millennium Run mock and the hydro mock run are cut off at the top in the same way, the hydro one more so. To get a better picture of the cosmic variance, Figure 7 shows the observations compared to hatched bands showing the $1 \sigma$ variation in the mock runs from the (a) DH and (b) MR simulations. It is clear from this that the one random Millennium run mock survey shown in figure 4 was worse than average at fitting the top of the curve, but it is also clear that the $\mathrm{DH}$ simulation is still better than the Millennium Run at fitting the observations because the band of Millennium run simulations are lower in this region. The place where the Millennium Run mocks and the DH mocks fail the worst is in the region $0.4<\nu<1.2$ where the observations are consistently shifted to the left with respect to the simulations.

We conclude that the simulations do an adequate job of representing the topology in all the variables except $\Delta \nu$, which for the SDSS data lies more than $2.5 \sigma$ away from either the MR or DH simulations. Of the $100 \mathrm{DH}$ mock surveys the one closest to the observations in terms of the four variables and their error bars is mock catalog 94 which has $\Delta \nu=-0.02, A=191, A_{v}=0.80, A_{c}=0.77$ which are OK in all except $\Delta \nu$ where it is still far off the observational value. In fact of the 100 mock $\mathrm{DH}$ simulations the two most negative in $\Delta \nu$ are between -0.05 and -0.04 while the observations show -0.08 . The four most negative of the 50 mock Millennium Run simulations are between -0.03 and -0.02 . Interestingly, the higher spatial resolution and semi-analytical modeling of the MR does not yield a better fit to the observed topology of large-scale structure than the dark-matter, halo-finding DH simulations.

\section{CONCLUSIONS}

The SDSS dataset has now become large enough that the topology of large-scale structure can be used for more detailed model testing. We find that the SDSS observations have a sponge-like median contour and follow fairly closely the genus curve expected from Gaussian random phase initial conditions predicted by inflation. We quantify departures from from this theoretical curve that provide key tests of models for galaxy formation, as represented by the several simulations that we examine.

The amplitude of the genus curve is in agreement with that predicted from the standard $\Lambda C D M$ model (with the WMAP parameters) with baryon oscillations included. If baryon oscillations were not included the fit to the amplitude would be significantly worse $(1.6 \sigma)$. The observed values of $A_{v}$ and $A_{c}$ are predicted well by both the MR and DH simulations. Both show the effects of non-linear gravitational evolution and biased galaxy formation. The Cen and Ostriker hydro simulations are consistent with the data, but their small volume gives them large error bars and they are currently not giving values closer to the observations than the $\mathrm{MR}$ or $\mathrm{DH}$ simulations.

The most notable feature of the observations is a meatball shift $\Delta \nu=-0.08$ showing a slight prominence of isolated high density regions over isolated voids. The SDSS Great Wall is one large connected isolated high density region and contributes to this effect, but the effect also shows up in the northern part of the SDSS which does not contain the Sloan Great Wall. If the Sloan Great Wall were entirely responsible for this result one might argue that it was produced by rare objects whose frequency of occurrence was determined by the power in the initial conditions at very large scales and that even larger simulations $>\left(1024 h^{-1} \mathrm{Mpc}\right)^{3}$ would be needed to properly test for this effect. But this is not the case. Negative values of $\Delta \nu=-0.08$ show up even in the part of the survey that does not include the SDSS Great Wall. Also, slice surveys of the MR simulation show great walls that look quite impressive - if not quite as dramatic as the Sloan Great Wall. The observed $\Delta \nu$ values are more than $2.5 \sigma$ away from the values found in the MR and the Dark Matter Halo simulations. This is a severe test for large N-body simulations and their heuristic galaxy formation scenarios because these were not tuned to account for topology.

The slight meatball shift seen in the observations has been noticed in previous observational samples with similar smoothing lengths $\left(6 h^{-1} \mathrm{Mpc}\right)$, being mentioned first by Gott et al. (1989). The large survey by Canaveses et al. (1998) of the IRAS galaxies looks Gaussian random phase on all larger smoothing lengths, but does have a slight meatball shift with a smoothing length of $5 h^{-1}$ Mpc. Hydrodynamic simulations suggest that early type galaxies should show more of a meatball shift than late type galaxies (Gott, Cen, \& Ostriker 1996) and this effect has already been observed in the equatorial slice of the SDSS in a 2D topology survey by comparing the relative meatball shift between red and blue galaxies (Hoyle et al. 2002).

As the SDSS Digital Sky Survey is completed, the gap between the northern and equatorial slices will be closed giving us one large continuous volume, where the fraction 


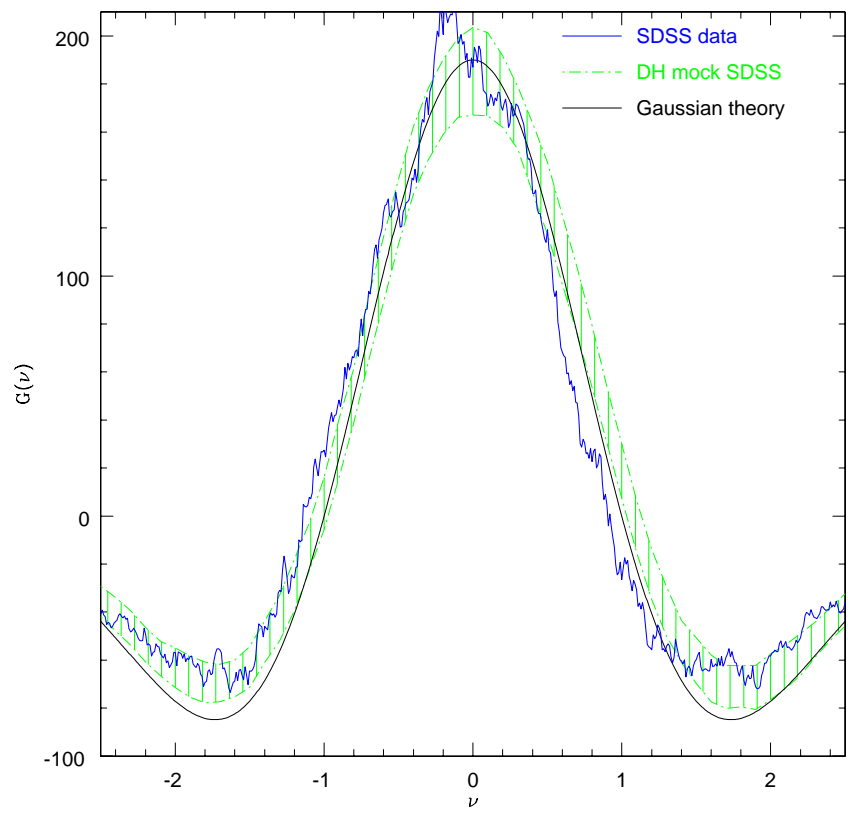

(a) $\mathrm{DH}$

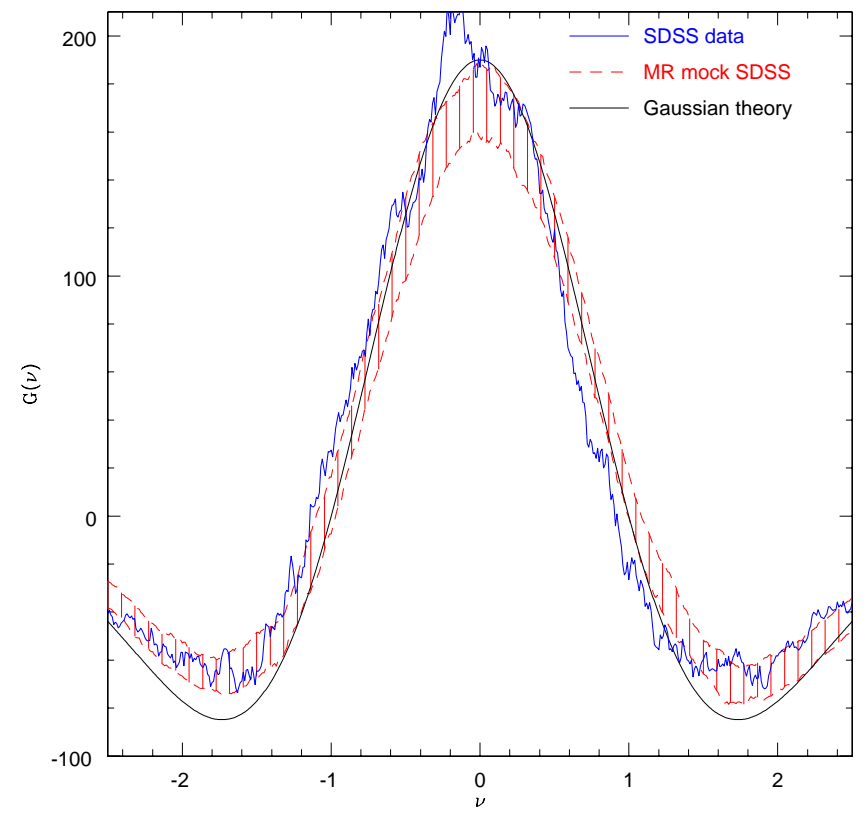

(b) MR

FIG. 7.- Genus curves with shaded $1 \sigma$ error regions for the (a) $100 \mathrm{DH}$ and (b) $50 \mathrm{MR}$ samples, compared with SDSS and Gaussian random phase.

of the sample one throws away because of closeness to the edge will be diminished. This will approximately double the effective volume of the sample and give us a still better test. Also, studies with smoothing lengths of $10 h^{-1}$ Mpc and $20 h^{-1} \mathrm{Mpc}$ will be possible with high precision allowing more direct tests of the Gaussian random phase hypothesis on scales where the galaxy formation effects are less important.

It would be interesting to see N-body simulations covering larger volumes which would have more power at large scales (because they would not be artificially cut off at the box size). This would make for more accurate modeling of the structure and frequency of occurrence of structures like the Sloan Great Wall (Gott et al. 2005).

The results here suggest that in order to account for the observed topology some changes in galaxy formation scenarios are called for. We look forward to improvements in the N-body simulations. Of particular interest is how well larger hydrodynamic simulations will perform when compared with larger samples, and whether there will be a convergence of predictions as both hydrodynamic and merger tree, and dynamical halo occupation methods are improved. Galaxy formation is not yet a solved problem in cosmology and the 3D topology offers a strong test of models which is independent of other measures.

\section{ACKNOWLEDGMENTS}

This paper has been supported by JRG's NSF Grant AST04-06713. MSV acknowledges support from NASA grant NAG-12243 and NSF grant AST-0507463. MSV thanks the Department of Astrophysical Sciences at Princeton University for its hospitality during sabbat- ical leave. MSV and CBP thank the Aspen Center for Physics, at which some of this work was completed. CBP acknowledges the support of the Korea Science and Engineering Foundation (KOSEF) through the Astrophysical Research Center for the Structure and Evolution of the Cosmos (ARCSEC), and through the grant R012004-000-10520-0. RC acknowledges support from NASA grant NNG05GK10G and NSF grant AST-0507521.

Funding for the SDSS and SDSS-II has been provided by the Alfred P. Sloan Foundation, the Participating Institutions, the National Science Foundation, the U.S. Department of Energy, the National Aeronautics and Space Administration, the Japanese Monbukagakusho, the Max Planck Society, and the Higher Education Funding Council for England. The SDSS Web Site is http: //www.sdss.org/

The SDSS is managed by the Astrophysical Research Consortium for the Participating Institutions. The Participating Institutions are the American Museum of Natural History, Astrophysical Institute Potsdam, University of Basel, Cambridge University, Case Western Reserve University, University of Chicago, Drexel University, Fermilab, the Institute for Advanced Study, the Japan Participation Group, Johns Hopkins University, the Joint Institute for Nuclear Astrophysics, the Kavli Institute for Particle Astrophysics and Cosmology, the Korean Scientist Group, the Chinese Academy of Sciences (LAMOST), Los Alamos National Laboratory, the Max-Planck-Institute for Astronomy (MPIA), the MaxPlanck-Institute for Astrophysics (MPA), New Mexico State University, Ohio State University, University of Pittsburgh, University of Portsmouth, Princeton Uni- 
versity, the United States Naval Observatory, and the University of Washington.

The Millennium Run simulation used in this paper was carried out by the Virgo Supercomputing Consortium at the Computing Centre of the Max-Planck Society in Garching. The semianalytic galaxy catalogue is publicly available at http://www.mpa-garching.mpg.de/galform/agnpaper and additional data are at http://www.mpa-garching.mpg.de/millennium

\section{REFERENCES}

Aarseth, S. J., Gott, J. R., \& Turner, E. L. 1979, ApJ, 228, 664

Abazajian, K., et al. 2004, AJ, 128, 502

Adelman-McCarthy, J.K. et al. 2006, ApJS, 162, 38

Adler, R. J. 1981, The Geometry of Random Fields (New York, Wiley)

Bertschinger, E. 1985, ApJS, 58, 1

Bhavsar, S. P., Gott, J. R., \& Aarseth, S. J. 1981, ApJ, 246, 656

Blanton, M. R., Lin, H., Lupton, R. H., Maley, F. M., Young, N., Zehavi, I., \& Loveday, J. 2003a, AJ, 125, 2276

Blanton, M. R., et al. 2003b, AJ, 125, 2348

Blanton, M. R., et al. 2005, AJ, 129, 2562

Canavezes, A., et al. 1998, MNRAS, 297, 777

Cen, R., Miralda-Escude, J., Ostriker, J. P., \& Rauch, M. 1994, ApJ, 437, L9

Cen, R., Nagamine, K., \& Ostriker, J.P., 2005, ApJ, 635, 86.

Cen, R., and Ostriker, K., 2006, in preparation

Choi, Y-Y, Park, C., \& Vogeley, M. S. 2006 ApJ, in preparation

Colley, W. N. 1997, ApJ, 489, 471

Colley, W. N., \& Gott, J. R. 2003, MNRAS, 344 (3), 686

Colley, W. N., Gott, J. R., \& Park, C. 1996, MNRAS, 281, L82

Colley, W. N., Gott, J. R., Park, C., Weinberg, D., \& Berlind, A. 2000, ApJ, 529,795

Coles, P. 1988, MNRAS, 234, 509

Croton, D.J., et al. 2006, MNRAS, 365, 11

Davis, M., Efstathiou, G., Frenk, C.S., \& White, S. D. M. 1985, ApJ, 292, 371

de Bernardis, P., et al. 2000, Nature, 404, 955

de Lapparent, V., Geller, M., \& Huchra, J. 1986, ApJ, 302, L1

Doroshkevich, G. 1970, Astrophysika, 6, 320

Einasto, J., Joeveer, M., \& Saar, E., 1980, MNRAS,193,353

Eisenstein, D. J., et al. 2001, AJ, 122, 2267

Fillmore, J., \& Goldreich, P., 1984, ApJ, 281, 9

Fukugita, M., Ichikawa, T., Gunn, J. E., Doi, M., Shimasaku, K., \& Schneider, D. P. 1996, AJ, 111, 1748

Geller, M. J., \& Huchra, J. P. 1989, Science, 246, 897

Gott, J. R., Cen, R. \& Ostriker, J. P. 1996, ApJ, 465, 499

Gott, J.R., Colley, W.N., Park, C-G., Park, C., Mugnolo, C. 2006, MNRAS (submitted)

Gott, J.R., Juric, M. et al., 2005, ApJ, 624, 463

Gott, J. R., Mao, S., Park, C., \& Lahav, O. 1992, ApJ, 385, 26

Gott, J. R., Melott, A. L. \& Dickinson, M. 1986, ApJ, 306, 341

Gott, J. R. \& Rees, M. J. 1975, Astronomy and Astrophysics, 45, 365

Gott, J. R. \& Turner, E.L. 1977, ApJ, 216, 357

Gott, J. R. \& Turner, E.L. 1979, ApJ, 232, L79

Gott, J. R., Turner, E.L., \& Aarseth, S. J. 1979, ApJ, 234, 13

Gott, J. R., Vogeley, M.S., Podariu, S. \& Ratra, B. 2001, ApJ, 549, 1

Gott, J. R., Weinberg, D. N. \& Melott, A. L. 1987, ApJ, 319, 1

Gott, J. R., et al. 1989, ApJ, 340, 625

Gott, J. R., et al. 1990, ApJ, 352, 1

Gunn, J.E. \& Gott, J. R. 1972, ApJ, 176,1

Gunn, J.E., et al. 1998, AJ, 116, 3040

Gunn, J.E., et al. 2006, AJ, 131, 2332

Guth, A.H. 1981, Phys. Rev. D., 23, 247

Hamilton, A.J.S., Gott, J.R., \& Weinberg, D.W. 1986, ApJ, 309, 1

Hernquist, L. Katz, N., Weinberg, D.H., \& Miralda-Escude, J. 1996, ApJ, 457, L51

Hikage, C., et al. 2002, Publ. Astron. Soc. Japan., 54, 707

Hikage, C., et al. 2003, Publ. Astron. Soc. Japan, 55(5), 911

Hogg, D. W., Finkbeiner, D. P., Schlegel, D. J., \& Gunn, J. E. 2001, AJ, 122, 2129
Hoyle, F., Vogeley, M.S., \& Gott, J. R. 2002, ApJ, 570, 44

Hoyle, F. et al. 2002, ApJ, 580, 663

Ivezic, Z. et al., AN, 325, 583

Kim, J., \& Park, C. 2006, ApJ, 639, 600

Kirshner, R. P., Oemler, A., Schechter, P. L., \& Shectman, S.A. 1981, ApJ, 248, L57

Kogut, A.J., et al. 1996, ApJ, 464, L29

Linde, A.D. 1983, Physics Letters, 129B, 177

Lupton, R.H., Gunn, J.E., Ivezic, Z., Knapp, G.R., Kent, S., \& Yasuda, N. 2001, in ASP Conf. Ser. 238, Astronomical Data Analysis Software and Systems X, ed. F. R. Harnden, Jr., F. A. Primini, \& H. E. Payne (San Francisco: ASP), 269

Lynden-Bell, D. et al. 1988, ApJ, 326, 19L

Matsubara, T. 1994, ApJ, 434, 43

Melott, A. L., Cohen, A. P., Hamilton, A. J. S., Gott, J. R., Weinberg, D. H. 1989, ApJ, 345, 618

Moore, B. et al., 1992, MNRAS, 256, 477.

Ostriker, J. P., \& Cowie, L. L. 1981, ApJ, 243, L127

Park, C. 1990, MNRAS, 242, 59P

Park, C. 1991, PhD Thesis, Princeton University

Park, C., Colley, W.N., Gott, J.R., Ratra, B., Spergel, D.N., \& Sugiyama, N. 1998, ApJ, 506,473

Park, C., \& Gott, J.R. 1991a, MNRAS, 249, 288

Park, C., \& Gott, J. R. 1991b, ApJ, 378, 457

Park, C., Gott, J.R. \& Choi, Y. J. 2001, ApJ, 553, 33

Park, C., Gott, J.R., Melott, A. \& Karachentsev, I. D. 1992, ApJ, 387,1

Park, C., Kim, J., \& Gott, J.R. 2005, ApJ, 633, 1

Park, C., et al. 2005, ApJ, 633, 11

Park, C., Choi, Y-Y, Vogeley, M.S., Gott, J.R., and Blanton, M.R. 2006, ApJ, submitted

Park, C.-G. 2003, MNRAS, 349, 313

Pier, J. R., Munn, J. A., Hindsley, R. B., Hennessy, G. S., Kent,

S. M., Lupton, R. H., \& Ivezic, R. 2003, AJ, 125, 1559

Peebles, P. J. E. 1974, ApJ, 189, L51

Peebles, P. J. E. 1978, A\&A, 68, 345

Perlmutter, S., et al. 1999, ApJ, 517, 565

Press, W. H., \& Schecter, P. 1974, ApJ, 187, 425

Reiss, A. G., et al. 1998, AJ, 116, 1009

Rhoads, J. E., Gott, J. R., \& Postman, M. 1994, ApJ, 421, 1

Richards, G. T., et al. 2002, AJ, 123, 2945

Schlegel, D. J., Finkbeiner, D. P., \& Davis, M. 1998, ApJ, 500, 525

Shectman, S. A., et al. 1996, ApJ, 470, 172

Smith, J. A., et al. 2002, AJ, 123, 2121

Spergel, D. N., et al. 2003, ApJS, 148, 175

Spergel, D. N., et al. 2006, ApJ, submitted, astro-ph/0603449

Springel, V., Frenk, C. S., \& White, S. D. M. 2006, Nature, 440, 1137

Springel, V., et al. 2005, Nature, 435, 629

Soneira, R. M., \& Peebles, P. J. E. 1978, AJ, 83, 845

Smoot, G. F., et al., 1994, ApJ, 437, 1

Stoughton, C., et al. 2002, AJ, 123, 485

Strauss, M. A., et al. 2002, AJ, 124, 1810

Tegmark, M., et al., 2004, ApJ, 606, 702

Tucker, D. et al. 2006, AN, in press

Vogeley, M. S., Park, C., Geller, M. J., Huchra, J. P., \& Gott, J. R. 1994, ApJ, 420, 525

York, D., et al. 2000, AJ, 120, 1579 